\title{
Aromatase inhibitors combined with aspirin to prevent lung cancer in preclinical models
}

\author{
Patricia A. Young, Richard J. Pietras \\ Department of Medicine, Division of Hematology-Oncology and Jonsson Comprehensive Cancer Center, UCLA David Geffen School of Medicine, \\ Los Angeles, CA, USA \\ Correspondence to: Patricia A. Young, MD. Department of Medicine-Hematology \& Oncology, UCLA David Geffen School of Medicine, 2020 Santa \\ Monica Blvd., Suite 600, Santa Monica, CA 90404, USA. Email: pyoung@mednet.ucla.edu. \\ Comment on: Stabile LP, Farooqui M, Kanterewicz B, et al. Preclinical Evidence for Combined Use of Aromatase Inhibitors and NSAIDs as \\ Preventive Agents of Tobacco-Induced Lung Cancer. J Thorac Oncol 2018;13:399-412.
}

Submitted Sep 17, 2018. Accepted for publication Sep 25, 2018.

doi: $10.21037 /$ tlcr.2018.09.17

View this article at: http://dx.doi.org/10.21037/tlcr.2018.09.17

\section{Introduction}

Lung cancer is the leading cause of cancer mortality worldwide (1). Although the incidence rate of lung cancer in women is lower than that of breast cancer, the 5 -year survival rate for lung cancer is less than $20 \%$ in North America and other developed countries, thereby making it the leading cause of cancer mortality among women $(2,3)$. More than $60 \%$ of lung cancer deaths in women are attributed to smoking (4), but a significant number of women afflicted with lung cancer are never smokers (5). Among non-smokers, lung cancer affects women disproportionately more than men, suggesting that sexrelated factors may in part play a role in lung cancer pathogenesis. A number of epidemiological, preclinical and early clinical studies provide convincing evidence on the role of estrogen signaling pathways in promoting lung cancer pathogenesis (6-16). Expression of estrogen receptors (ER $\alpha$ and $\mathrm{ER} \beta$ ), progesterone receptors (PR), and/or aromatase, a key enzyme in estrogen biosynthesis, is often reported in human non-small cell lung cancers (NSCLCs) $(6,7,10,15)$. Importantly, high expression of tumor aromatase enzyme is a poor prognostic biomarker in postmenopausal women afflicted with NSCLC (10). In preclinical models in vitro and in vivo, estradiol-17 $\beta$ stimulates the proliferation of NSCLC $(6,8,11)$. Selective ER downregulators (such as fulvestrant) and aromatase inhibitors (AIs; such as anastrazole, letrozole, exemestane) that block estrogen synthesis inhibit lung tumor growth (7-9) and prevent lung tumorigenesis in preclinical models (13). Of note, estrogen synthesis and/or activity among non-tumor cells in the tumor microenvironment such as selected immune and inflammatory cells (e.g., lymphocytes, macrophages) is also reported and may play a role in modulating tumor progression in vivo $(13,17)$. Hence, it is conceivable that inflammation due to underlying inflammatory conditions or local inflammation triggered by chronic tobacco exposure in the lungs may lead to up-regulation of estrogen signaling in tumor-infiltrating immune cells that may then contribute to lung tumor progression.

\section{Results}

In their report published in the fournal of Thoracic Oncology from March 2018, Stabile et al. (18) evaluated the combination of AIs and non-steroidal anti-inflammatory drugs (NSAIDs) on lung tumor size and growth along with the inflammatory pathways for NSCLC prevention. The group hypothesized that inhibiting tissue aromatase activity with an $\mathrm{AI}$ and simultaneous reduction of inflammation with a NSAID may be an effective strategy to suppress estrogen signaling as well as estrogen-induced pro-inflammatory cytokines and macrophage recruitment that serve to amplify pro-tumorigenic effects of estrogens $(13,17,18)$. This notion is supported by several studies that have reported a link between NSAID use (aspirin and ibuprofen) and a reduced risk of lung cancer (19). In addition, NSAID use is independently shown to modulate aromatase activity and serum total estradiol levels in postmenopausal women $(18,20)$. 
The investigators used an established tobacco carcinogen [4-(methylnitrosoamino)-1-(3-pyridyl)-1-butanone (NNK)]-induced lung tumor mouse model to determine whether an AI combined with aspirin results in greater lung tumor prevention effects than single agent treatment. The NNK tumor model was intended to represent ex-smoking postmenopausal women as high-risk individuals for the development of lung cancer. Female ovariectomized FVB/ $\mathrm{N}$ mice (6 weeks of age) were exposed to NNK ( $3 \mathrm{mg}$ per intraperitoneal injection; twice/week) for 4 weeks. Therapy was initiated after 5 weeks to allow for lung preneoplasia development. Androstenedione, the substrate for aromatase, at $0.1 \mathrm{mg}$ was administered subcutaneously three times per week throughout the experiment. Anastrazole $(0.1 \mathrm{mg} / \mathrm{kg})$ orally alone, aspirin $(25 \mathrm{mg} / \mathrm{kg})$ orally alone, or the combination of anastrazole and aspirin, and vehicle control groups were treated 5 times per week for 8 weeks. In addition, a short-term 2 -week treatment exposure experiment was done to evaluate cytokine expression in the lungs and blood prior to tumor formation. Mice were sacrificed after 8 weeks of treatment for AI/NSAID studies followed by formalin-inflation of lungs and collection of blood, with surface lung tumors later counted under a dissecting microscope. The results indicate that dual treatment of mice with anastrazole $(0.1 \mathrm{mg} / \mathrm{kg} /$ day dosing $)$ and aspirin $(25 \mathrm{mg} / \mathrm{kg} /$ day $)$ after NNK exposure elicited significantly fewer and smaller lung tumors as compared to single-agent treatments in control animals.

Moreover, these antitumor effects with combination treatment were accompanied by marked reduction in circulating estradiol-17 $\beta$ and IL-6 levels. In addition, they found a significant decrease in the lung tumor proliferation biomarker Ki-67, as well as reduced downstream phosphorylation of tumor mitogen-activated protein (MAP) kinase and signal transducer and activator of transcription 3 (STAT3) and reduced $I L-17 A$ expression. Notably, tumorinfiltrating macrophage levels were also decreased in the tumor microenvironment. The investigators noted that pre-neoplasia arising after combination treatment with $\mathrm{AI}$ and aspirin showed the lowest level of the tumor stem/ progenitor cell marker Sox-2 expression and suggest that this finding connotes an inhibitory effect on proliferative capacity in the airways by blocking both estrogen signaling and inflammation processes. Anastrozole combined with ibuprofen instead of aspirin also showed enhanced antitumor effects but these effects were less pronounced.

Using a different murine model to assess effects of estrogen exposure in vivo, the investigators treated male mice (chosen to minimize the effects of circulating estradiol found in females) with NNK for 4 weeks and simultaneously administered estrogens or placebo control in the drinking water. The male mice were then sacrificed after 4 weeks for studies as above, with the results showing higher levels of infiltrating pulmonary macrophages and inflammatory biomarkers as compared to controls. Thus, these findings using an ER agonist offer confirmation of a direct effect of estrogen signaling on inflammation in the tumor microenvironment.

\section{Evaluation of the evidence}

The authors provide a comprehensive statistical analysis suggesting a significant decrease in both the number and size of tumors in the combination group compared to either single agent therapy and vehicle control. Furthermore, to provide a potential mechanism for this result, cytokine levels were measured. When evaluating IL-6 levels, a notable decrease between combination therapy and vehicle control is noted $(\mathrm{P}=0.0004)$; however, comparing combination treatment with either single agent is insignificant. The variability in the IL-6 level in the control group is also noted by the authors, which is not explained by tumor burden alone. A short term (2-week) experiment demonstrated decreased IL-6 levels after 2 weeks of individual or combination therapy. No statistical analysis is provided to evaluate whether treatment is the result of these decreases in IL-6 levels.

The experiments reported here suggest the role of estrogen driven regulation of the inflammatory lung microenvironment, similar to observations in breast cancer. The limitations of these studies are the in vivo nature of these preclinical experiments, but the authors appropriately point out the necessary studies that will be needed to evaluate the efficacy of this treatment approach in clinical practice. The series of experiments performed in this original article provide stimulating evidence that combination AI and NSAID therapy may reduce lung cancer incidence among high risk postmenopausal women with inflammatory lung disease.

\section{Conclusions}

This report presents new data to support the idea that use of aromatase inhibitors alone and combined with NSAIDs can help to prevent lung tumorigenesis as assessed in a murine preclinical model. By comparison with the known 
antitumor and cancer preventive activity of aromatase inhibitors in breast cancer $(21,22)$, the current report (18) and results of recent clinical trials using antiestrogens (15) and aromatase inhibitors (16) (NCT01664754) in patients with advanced lung cancer suggest that dual targeting of estrogen and inflammatory signaling pathways may have benefit for NSCLC prevention. A phase II therapeutic study of adding AI exemestane therapy in post-menopausal women with advanced NSCLC who are progressing while on treatment with an immune checkpoint antibody is an ongoing study (NCT02666105). As shown by the investigators, combination of aromatase inhibitors with NSAIDs reduces circulating levels of estrogens and proinflammatory cytokines, and macrophage recruitment in the lung tumor microenvironment following tobacco carcinogen exposure. This treatment strategy could be especially effective in women at increased risk for lung cancer who also have underlying pulmonary inflammatory disease.

\section{Acknowledgements}

Research funding provided by Gateway for Cancer Research (RJ Pietras, PA Young).

\section{Footnotes}

Conflicts of Interest: R.J.P. has served previously as a consultant for Astra-Zeneca and Pfizer Pharmaceuticals. The other author has no conflicts of interest to declare.

\section{References}

1. Siegel RL, Miller KD, Jemal A. Cancer Statistics, 2017. CA Cancer J Clin 2017;67:7-30.

2. Martín-Sánchez JC, Lunet N, González-Marrón A, et al. Projections in Breast and Lung Cancer Mortality among Women: A Bayesian Analysis of 52 Countries Worldwide. Cancer Res 2018;78:4436-42.

3. Stewart B, Wild C, editors. World cancer report 2014. Lyon: International Agency for Research on Cancer; 2014.

4. World Health Organization. WHO global report: mortality attributable to tobacco. Geneva: WHO; 2012.

5. Subramanian J, Govindan R. Lung cancer in never smokers: a review. J Clin Oncol 2007;25:561-70.

6. Stabile LP, Davis AL, Gubish CT, et al. Human nonsmall cell lung tumors and cells derived from normal lung express both estrogen receptor alpha and beta and show biological responses to estrogen. Cancer Res 2002;62:2141-50.

7. Weinberg OK, Marquez-Garban DC, Fishbein MC, et al. Aromatase inhibitors in human lung cancer therapy. Cancer Res 2005;65:11287-91.

8. Stabile LP, Lyker JS, Gubish CT, et al. Combined targeting of the estrogen receptor and the epidermal growth factor receptor in non-small cell lung cancer shows enhanced antiproliferative effects. Cancer Res 2005;65:1459-70.

9. Hershberger PA, Vasquez AC, Kanterewicz B, et al. Regulation of endogenous gene expression in human nonsmall cell lung cancer cells by estrogen receptor ligands. Cancer Res 2005;65:1598-605.

10. Mah V, Seligson DB, Li A, et al. Aromatase expression predicts survival in women with early-stage non small cell lung cancer. Cancer Res 2007;67:10484-90.

11. Márquez-Garbán DC, Chen HW, Goodglick L, et al. Targeting aromatase and estrogen signaling in human non-small cell lung cancer. Ann N Y Acad Sci 2009;1155:194-205.

12. Chlebowski RT, Schwartz AG, Wakelee H, et al. Oestrogen plus progestin and lung cancer in postmenopausal women (Women's Health Initiative trial): a post-hoc analysis of a randomised controlled trial. Lancet 2009;374:1243-51.

13. Stabile LP, Rothstein ME, Cunningham DE, et al. Prevention of tobacco carcinogen-induced lung cancer in female mice using antiestrogens. Carcinogenesis 2012;33:2181-9.

14. Chu SC, Hsieh CJ, Wang TF, et al. Antiestrogen use in breast cancer patients reduces the risk of subsequent lung cancer: A population-based study. Cancer Epidemiol 2017;48:22-8.

15. Garon EB, Siegfried JM, Stabile LP, et al. Randomized phase II study of fulvestrant and erlotinib compared with erlotinib alone in patients with advanced or metastatic non-small cell lung cancer. Lung Cancer 2018;123:91-8.

16. Young PA, Marquez DC, Noor ZS, et al. IASLC 19th World Conference on Lung Cancer. Available online:https://wclc2018.iaslc.org/

17. Rothenberger NJ, Somasundaram A, Stabile LP. The Role of the Estrogen Pathway in the Tumor Microenvironment. Int J Mol Sci 2018;19.

18. Stabile LP, Farooqui M, Kanterewicz B, et al. Preclinical Evidence for Combined Use of Aromatase Inhibitors and NSAIDs as Preventive Agents of Tobacco-Induced Lung Cancer. J Thorac Oncol 2018;13:399-412.

19. Hochmuth F, Jochem M, Schlattmann P. Meta-analysis of 
aspirin use and risk of lung cancer shows notable results. Eur J Cancer Prev 2016;25:259-68.

20. Hudson AG, Gierach GL, Modugno F, et al. Nonsteroidal anti-inflammatory drug use and serum total estradiol in postmenopausal women. Cancer Epidemiol Biomarkers Prev 2008;17:680-7.

21. Goss PE, Ingle JN, Alés-Martínez JE, et al. Exemestane

Cite this article as: Young PA, Pietras RJ. Aromatase inhibitors combined with aspirin to prevent lung cancer in preclinical models. Transl Lung Cancer Res 2018;7(Suppl 4):S373-S376. doi: 10.21037/tlcr.2018.09.17 for breast-cancer prevention in postmenopausal women. N Engl J Med 2011;364:2381-91.

22. Cuzick J, Sestak I, Forbes JF, et al. Anastrozole for prevention of breast cancer in high-risk postmenopausal women (IBIS-II): an international, double-blind, randomised placebo-controlled trial. Lancet 2014;383:1041-8. 